\title{
Roger Acher (1923-2020)
}

Nous avons appris le décès de notre confrère, le Professeur Roger Acher, le 15 juin dernier dans sa quatrevingt-dix-septième année. C'était un des derniers pionniers de la neuroendocrinologie française (Figure 1).

Comme beaucoup de jeunes gens de sa génération, Roger Acher a d'abord dû traverser une époque troublée (http://www.francaislibres.net/liste/fiche.php?index= 50372). Né le 10 décembre 1923 à Héliopolis en Égypte, sa famille s'installe ensuite à Contes, un village de l'arrièrepays niçois. Après avoir obtenu un baccalauréat en Mathématiques et un en Philosophie à Nice, il entame des études d'agronomie à l'École Coloniale d'Agriculture de Tunis (ECAT). Le 7 mai 1943, Roger Acher est présent lorsque la ville est libérée par les Anglais de Montgomery et la «Force L » du Général Leclerc. Le 15 mai, il s'engage au $3^{\mathrm{e}}$ Régiment d'Artillerie Coloniale, composé de Français Libres de la Force L. Fin août 1944, après deux mois d'entraînement en Libye, il est versé au XI/64 RADB, un des trois régiments d'artillerie de la $2^{\mathrm{e}} \mathrm{DB}$. Affecté à la $33^{\mathrm{e}}$ batterie, il fait toute la guerre comme pointeur du canon automoteur Neptune. Meilleur pointeur de son régiment, il participe à toutes les batailles de la $2^{\mathrm{e}} \mathrm{DB}$ : débarquement à Utah Beach, combats de Normandie (notamment à Argentan, où sa pièce tire sans discontinuer pendant trois jours et deux nuits), libération de Paris, combats de Lorraine (Chatel, Nomexy, prise de Baccarat), puis campagne d'Alsace, avec les durs combats dans la neige au sud de Strasbourg pour contenir la poussée allemande de l'offensive «Nordwind» à la mi-décembre 1944, et réduction de la poche de Colmar. Enfin, Roger Acher entre dans Berchtesgaden avec sa batterie le 5 mai 1945 , pratiquement trois ans - jour pour jour - après son engagement. La veille, dans la vallée d'Inzel, le Neptune avait tiré les derniers obus de la $2^{\mathrm{e}} \mathrm{DB}$ lors de la Seconde Guerre mondiale. Roger Acher a la fierté de défiler sur les Champs-Élysées sur le Neptune le 14 juillet 1945. De retour en France, il apprend l'arrestation par la Gestapo de ses parents à leur domicile de Contes, sur dénonciation du voisinage, le 15 septembre 1943, une semaine après l'occupation de Nice par les Allemands. Tous deux furent déportés et assassinés à Auschwitz en octobre 1943 (convoi 60).
Roger Acher obtient son diplôme d'Ingénieur Agronome de l'ECAT en 1946 et reprend ses études en Chimie et Biologie à Paris. Boursier de recherche dans le laboratoire de Claude Fromageot en 1947, il adhère au concept, révolutionnaire à l'époque, de la nature peptidique des neurohormones hypothalamiques et s'engage dans la course à la structure des hormones neurohypophysaires. En 1895, Oliver et Schafer avaient démontré qu'une substance d'origine hypophysaire augmentait la pression sanguine. En 1906, Henry Dale avait montré, chez la chatte gravide, les propriétés contractiles d'une autre substance produite par l'hypophyse. À partir de 1927, la pitossine et la pitocine étaient séparées à partir d'extraits hypophysaires et le second principe était commercialisé sous le nom d' «Oxytocin» et prescrit pour le travail de l'accouchement. En 1953, Vincent du Vigneaud publie la structure nonapeptidique de l'ocytocine, ce qui lui vaut le prix Nobel de chimie deux ans plus tard. Parallèlement, dans son laboratoire du boulevard Raspail à Paris, Roger Acher découvre la structure de la vasopressine (Acher \& Chauvet, 1953) et montre que la protéine, dite de Van Dyke, qui paraît posséder une double activité ocytocique et vasopressique peut être séparée en hormones libres et une protéine porteuse qu'il nomme neurophysine (Acher et al., 1956). Sa notoriété naissante lui permet alors de rejoindre en tant que «Rockefeller fellow» le laboratoire de du Vigneaud à l'université Cornell à New York. Il y adapte la technique de chromatographie échangeuse d'ions qui facilite la purification des hormones neurohypophysaires (Light et al., 1957). De retour en France, il est nommé professeur de chimie biologique à la Faculté des Sciences de Marseille. Un an plus tard il revient à Paris, toujours boulevard Raspail, comme Professeur de chimie biologique à la Faculté des Sciences qui deviendra l'Université Paris VI. À partir de ce moment, Roger Acher s'intéresse principalement à l'évolution phylogénétique des neurohormones hypophysaires. Par de nombreuses études de biochimie comparée, il montre, en particulier, l'identité de l'ocytocine purifiée à partir du réseau neuronal de l'hydre, de l'intestin du rat, et du cerveau humain (Acher, 1985), contrastant avec la variabilité phylogénétique des molécules apparentées à 


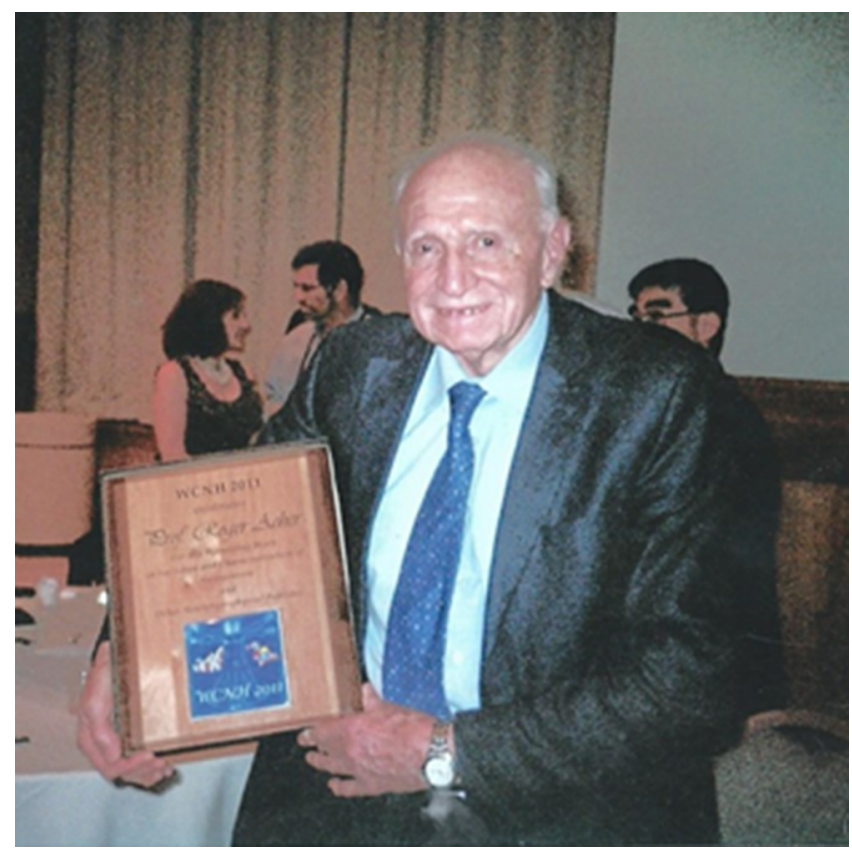

Roger Acher récipiendaire du prix du World Congress on Neurohypophysial Hormones 2011 (WCHN 2011) pour son travail pionnier dans l'isolation et la caractérisation de la vasopressine et d'autres peptides neurohypophysaires.

la vasopressine, des amphibiens aux marsupiaux, finalement résumée dans son dernier article indexé dans PubMed (Acher, 2002).

Jusqu'à la fin, Roger Acher a été un membre assidu des réunions de la Société de Biologie, et ses nombreuses questions ont toujours animé nos discussions. Son enthousiasme pour la Science était toujours le même. Comme nous l'a indiqué sa nièce, Francine Acher, ellemême directrice de recherche au CNRS et responsable d'une équipe au sein du Laboratoire de Chimie et Biochimie Pharmacologiques et Toxicologiques à l'Université Paris Descartes, Roger Acher, malgré son grand âge, était toujours abonné à de nombreuses revues scientifiques dont Science, Nature et Biologie Aujourd'hui.

Un bel exemple à suivre.

Jacques EPELBAUM*

UMR CNRS/MNHN 7179, Mécanismes Adaptatifs et Évolution, 1 avenue du Petit Château, 91800 Brunoy, France

\footnotetext{
* jacques . epelbaum@inserm.fr
}

\section{Références}

Acher, R., Chauvet, J. (1953). La structure de la vasopressine de bœuf. Biochim Biophys Acta, 12, 487-488.

Acher, R., Chauvet, J., Olivry, G. (1956). Sur l'existence éventuelle d'une hormone unique neurohypophysaire. 1. Relations entre l'ocytocine, la vasopressine et la protéine de van Dyke extraite de la neurohypophyse de bœuf. Biochim Biophys Acta, 2, 421-427.

Acher, R. (1985). The nonmammalian mammalian transition through neurohypophysial peptides. Peptide, Suppl. 3, 309314.

Acher, R. (2002). L'homéostase hydrique dans le vivant : organisation moléculaire, réflexes osmorégulateurs et évolution. Ann Endocrinol (Paris), 63, 197-218.

Light, A., Acher, R., du Vigneau, V. (1957). Ion exchange chromatography of purified posterior pituitary preparations. J Biol Chem, 228, 633-641. 